\title{
Analysis of new approaches used in portfolio optimization: a systematic literature review
}

\author{
Danilo Alcantara Milhomem ${ }^{\mathrm{a} *}$ (D), Maria José Pereira Dantas ${ }^{\mathrm{a}}$ \\ aPontifícia Universidade Católica de Goiás, Goiânia, GO, Brasil \\ *daniloalcantaramilhomem@gmail.com
}

\begin{abstract}
Paper aims: To do a comprehensive review of the exact and heuristic methods, software/programming languages, constraints, and types of analysis (technical and fundamental) used to solve the portfolio optimization problem.

Originality: The paper presents a useful discussion on aspects of portfolio optimization, both for researchers and investors and for finance professionals.
\end{abstract}

Research method: A systematic literature review was performed, and the articles were compiled according to pre-established criteria/filters.

Main findings: A point of attention should be given to the input data of optimization models. Depending on the degree of the estimation error of these input parameters, the optimization results may be lower than the results of the $1 / \mathrm{N}$ trading strategy.

Implications for theory and practice: Robust optimization, Fuzzy logic, and prediction are examples of techniques used to reduce estimation errors. At the end of the article are pointed out trends and some gaps for future work.

Keywords

Portfolio selection. Heuristics. Constraints. Stock market.

How to cite this article: Milhomem, D. A., \& Dantas, M. J. P. (2020). Analysis of new approaches used in portfolio optimization: a systematic literature review. Production, 30, e20190144.

Received: Nov. 27, 2019; Accepted: June 15, 2020.

\section{Introduction}

Portfolio Optimization consists of determining a set of assets, and their respective portfolio participation weights, which satisfy the investor concerning the combination of risk-return binomial. Given this problem, Markowitz (1952), proposed the Mean-Variance (MV) model. In the model, the expected return is given by the average of the historical data of the stock's return, and the risk is calculated by the variance of these returns. The main idea of the MV model is to deal with the returns of individual assets as random variables and to adopt the value of expected return and variance in order to quantify the return and investment risk, respectively (Zhang et al., 2018).

If the customer wants to minimize the risk for a given value of fixed return, then the mathematical model that will select the stocks is given by (for a given level of risk, maximizing returns is also its equivalent model):

$$
\begin{gathered}
\text { MinimizeVar }\left[\xi_{1} x_{1}+\xi_{2} x_{2}+\cdots+\xi_{n} x_{n}\right] \\
\text { s.t.E }\left[\xi_{1} x_{1}+\xi_{2} x_{2}+\cdots+\xi_{n} x_{n}\right] \geq \alpha \\
x_{1}+x_{2}+\cdots+x_{n}=1 \\
x_{i} \geq 0, i=1,2, \ldots, n,
\end{gathered}
$$


where $E$ represents the operation of expected value; $V a r$ is the variance operator; and $x_{i}$ is the proportion of the total amount available for investment applied in the stock $i, \xi_{1}$ represents the random return for the ith stock (Zhang et al., 2018).

The objective function of minimizing the risk is given by Equation 1, in Equation $2 \alpha$ represents the minimum return value that the investor can accept, the budget constraint is given by the Equation 3 and indicates that all available money must be invested, and the non-negativity constraint it means that selling short are not accepted.

Merton (1971) approaches the portfolio optimization problem from the perspective of how to systematically build and analyze optimal dynamic models of continuous-time under uncertainty. According to the author, the main advantage of the continuous-time model comparing to models that consider the discrete-time is the fact that it contemplates only two types of stochastic processes: functions of Brownian movements and Poisson processes. Thus, the number of parameters in the problem is reduced, which allows taking full advantage of the huge amount of written literature about these processes.

In Uryasev (2000), Conditional-Value-at-Risk (CVaR) is proposed as a risk measure of the portfolio, and therefore optimization would be on top of that metric. The $C V a R$ is derived from the weighted average of "extreme" losses in the tail of the distribution of possible returns, in addition to the cutoff point of the Value at Risk (VaR). One of the advantages of $\mathrm{CVaR}$ is that it can be minimized efficiently using LP (Linear Programming) techniques.

Another approach to the portfolio problem is robust optimization, which, according to Fabozzi et al. (2007), is the act of incorporating into the model, through mathematical techniques, the uncertainty generated by the errors of estimation of the parameters used in optimization. According to Pflug \& Wozabal (2007) and Fabozzi et al. (2007), robust optimization offers as an advantage the fact that modifications in the mathematical model do not change the characteristics of the problem, which remains a problem of quadratic programming.

DeMiguel et al. (2009a), Pflug et al. (2012) and Behr et al. (2013) report that the uniform investment strategy (Naive diversification or $1 / \mathrm{N}$ ) is rational the greater the degree of uncertainty about the risk/return distribution is, and this fact is supported by several experimental studies which demonstrate that this kind of strategy tends to be better in environments of extreme uncertainty, and is usually a good strategy for risk-averse investors. DeMiguel et al.(2009a) show in what situations Naive Diversification is preferred to optimization, and the results by Behr et al. (2013) corroborate those of DeMiguel et al. (2009a), showing that the optimization strategies are not unanimously better than the $1 / \mathrm{N}$ strategy, from the perspective of the estimation error outside the sample.

Sparse and stable optimization is an important advance for mean-variance portfolio selection. In general, DeMiguel et al. (2009b), Brodie et al. (2009), Fan et al. (2012), Behr et al. (2013) and Dai \& Wen (2018) defends the idea of taking into account weights that can assume negative or positive values (Long and Short positions). The idea is to stabilize the weights of the assets to reduce the estimation error using the Mean-Variance model with the addition of the restriction that the norm of the portfolio weight vector is less than a certain limit. The sum of the weights can be less than or equal to 1 (1-norm). In addition, the model consists of admitting negative values (Short), but normalization brings them to the positive side. Furthermore, the threshold of the sum of the weights can be greater than or equal to 1. This simple change brought better results in terms of Sharpe than strategies present in the literature.

Some authors reviewed the Portfolio optimization methods. For example: Hu et al. (2015), studied the application of evolutionary computation in the discovery of rules in algorithmic trading for shares; Ertenlice \& Kalayci (2018), conducted a swarm intelligence research for portfolio optimization, discussing algorithms and applications; Zhang et al. (2018), reviewed the portfolio optimization problem with the Markowitz mean-variance structure; Mansini et al. (2014), reexamined over 20 years of portfolio optimization based on operational research.

The contribution of this work lies in the fact that no article has been found in the literature, which broadly and jointly encompasses the items: heuristic and exact methods, realistic constraints, evaluated markets, types of stock analysis, and software/Programming language.

Given the importance of the portfolio optimization theme and the scope of related matters, this study has as purpose to identify, by means of a Systematic Literature Review (SLR), the main methods, tools and techniques of portfolio optimization, real-world constraints, and to analyze how the applications of this set were changing over the years.

This article is organized as follows: Section 2 presents the method through which state of the art was sought in the literature on portfolio optimization; in Section 3, the results are presented; in Section 4, the results are discussed, and the research questions are answered. Moreover, the conclusions are shown in Section 5. 


\section{Research method}

This study is categorized as a configurative review, as it explored the theme defined with qualitative data and data gathered from more heterogeneous primary studies. The RSL steps performed were based on Morandi \& Camargo (2015) and complemented, according to Bastos et al. (2017), Furlan \& Laurindo (2017), and Yu et al. (2018). The stages of the article's development are documented in the protocol for Systematic Literature Review (Table 1).

\subsection{Research questions}

The Research Questions in Table 1 guided the authors on the aspects that should be observed in reading the compiled articles. Discussion/answers to Research Questions are made in Section 4.

\subsection{Inclusion criteria and exclusion criteria}

The papers selected were those published in journals in the period between 2014 and 2018. Other than this criterion, Figure 1 has the inclusion or exclusion filters for the papers. Search strings are defined in Table 1.

The papers were selected according to their approach regarding the Portfolio optimization theme and their potential relevance to solving the research questions shown in Table 1.

\subsection{Deviations in the protocol}

Some research steps were changed regarding what was proposed:

a) The use of the impact factor in journal evaluations instead of the Qualis classification;

b) Inclusion of analysis on the data used to test the algorithms or models (whether they were real or of instances);

c) Inclusion of VosViewer software for macro analysis of the articles found and their references to classical authors.

Table 1. Protocol for Systematic Literature Review.

\section{PROTOCOL FOR SYSTEMATIC LITERATURE REVIEW: SEARCH STRATEGY}

Central Theme: The Central Theme is Portfolio Optimization. This theme will be approached in an open manner with the objective of finding the greatest number of academic and scientific publications.

Conceptual Framework: Portfolio Optimization is a very important topic in the stock market area. Since the creation of the Mean-Variance Model by Markowitz (1952), several studies have been developed in order to improve the model/create more robust analysis methods through the insertion of realistic constraints in the model. This review has the purpose of identifying the studies that include the theme, the tools, and methods for optimizing Portfolio.

Background:

Scope:

Theoretical currents: Languages:

Research Questions:

Review Strategy:

Inclusion Criteria

Exclusion Criteria

Search terms:
Diversification and optimization of an investment portfolio.

The Search period will be between 2014 and 2018, with the intention of finding the most relevant publications published in the last five years.

Portfolio Optimization.

Both the search terms and the papers will be in English.

Question 1 - Which key methods, tools, or techniques are used in portfolio optimization?

Question 2 - Which realistic constraints are used?

Question 3 - What type of analysis is done regarding the stock: fundamental, technical, or mixed (fundamental and technical)?

Question 4 - Which software/programming language are used?

\section{(X) Configurative () Aggregative}

The papers should contain the latest discoveries about portfolio optimization models as well as realistic constraints, analysis types, and algorithms.

Duplicate papers

1st search: "Optimization" AND "portfolio" AND "algorithms" AND "return" AND "risk";

2nd search: "Optimization" AND "portfolio" AND "algorithms" AND "return" AND "risk";

3rt search: "Portfolio Optimization" AND "stock market" AND "Markowitz";

4th search: "stock market" AND "Portfolio selection" AND "portfolio optimization";

5th search: "Portfolio Optimization" AND "stock market" AND "financial".

Search Sources 


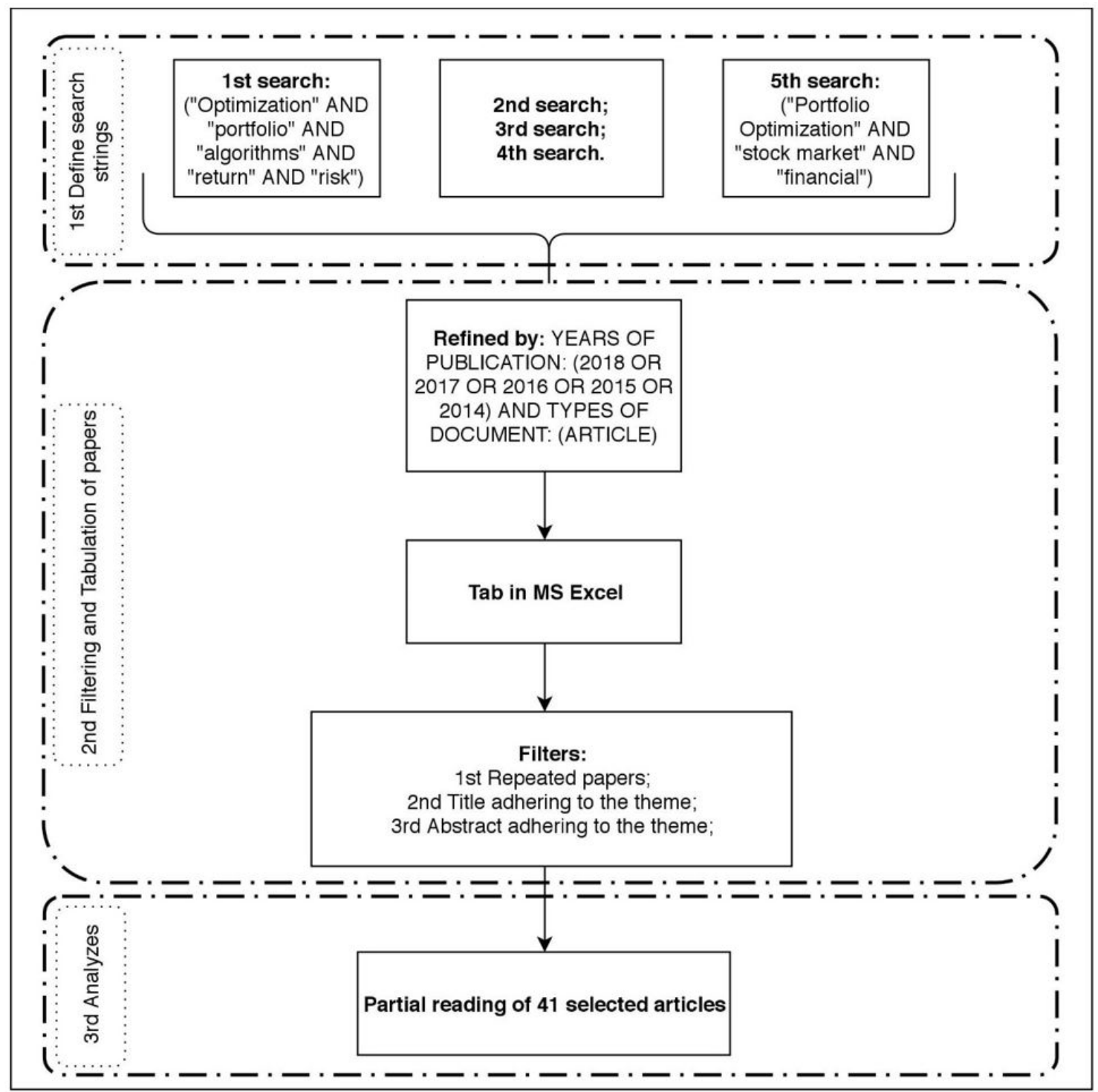

Figure 1. Inclusion Criteria and Exclusion criteria.

\section{Results}

The following is a macro view of the results of the search for papers on the portfolio optimization theme. By combining Search terms (Table 1) into a single set of search strings ("Optimization" AND "portfolio" OR "Portfolio Optimization" OR "Portfolio selection" AND "algorithms" AND "return" AND "risk" AND "stock market" AND "Markowitz" AND "financial"), and refinement for works written in the English language, was found 2137 papers on portfolio optimization. With these papers, some analyzes were performed with the aid of VosViewer software. A survey of the main words used in the titles of the articles was performed, whose mapping can be seen in Figure 2 .

Note in Figure 2, the presence of terms such as transaction costs, risk management, dynamic programming, and robust optimization. Besides, the terms related to portfolio optimization have also been identified: genetic algorithms, multi-objective optimization, and stochastic optimization. These terms give clues about the lines of research explored by the articles found.

In Figure 3, the main countries where the papers were published are shown. China and the USA appear as the two countries that publish the most on the topic under study. Followed by Germany, France, Canada, and England. Brazil appears on the map with a considerable number of publications on the subject. 


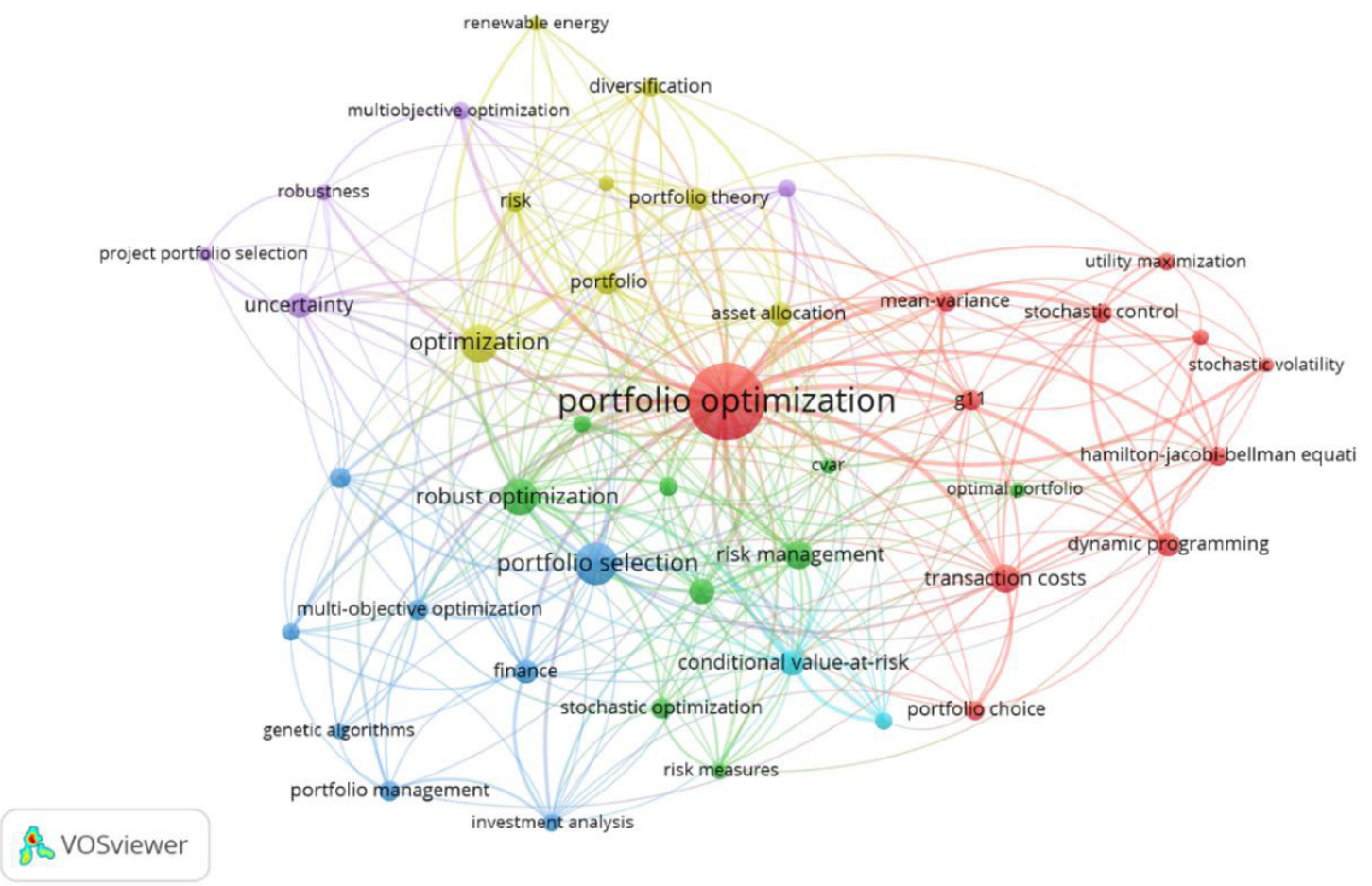

Figure 2. Map of keywords contained in the title of the publications.

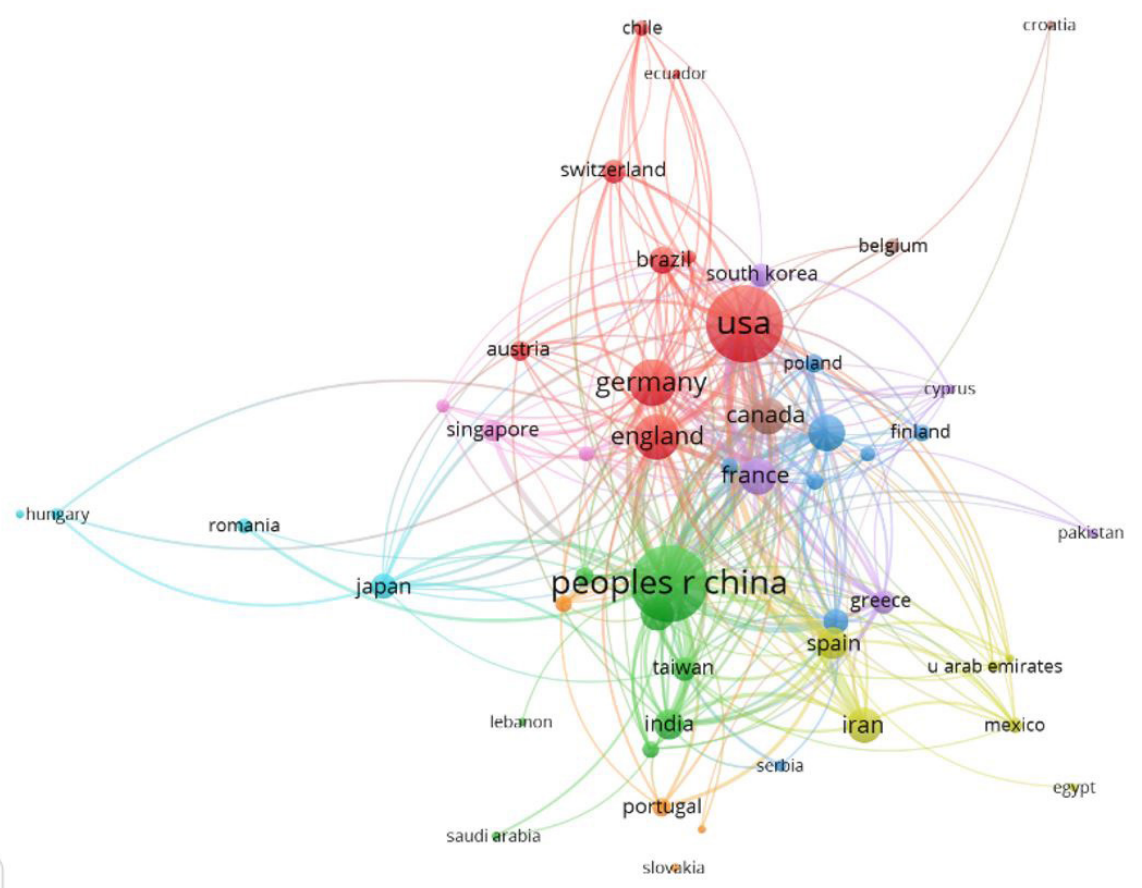

Figure 3. Country mapping in terms of publication. 
In Figure 4, the mapping of the most cited authors in the studies researched is shown. The author is identified on the map, along with the year of publication of the aforementioned work and the place of publication.

Markowitz (1952) appears as the most cited author, evidencing that the Medium Variance model is still widely discussed in the financial market and the universe of portfolio optimization. In addition to Markowitz, authors such as Zadeh (1965), DeMiguel et al. (2009a), Uryasev (2000), and Merton (1971) wrote important articles on the topic portfolio optimization, and all of these authors continue to be widely cited.

\subsection{Research results}

Table 2 shows the journals where the analyzed papers were published. This table presents two important information: the Impact factor (internationally recognized index, used to measure the journal's relevance and scope in Publications), and the number of papers per journal. Table 2 data show that the selected papers come from excellent journals.

As a result of the search process, a total of 41 unique studies (without duplicity) were identified after the application of the searches and the inclusion and exclusion criteria/filters. In Table 3 and Table 4, there were only the papers on which the partial reading was carried out, except for the bibliographic review papers (Ertenlice \& Kalayci, 2018; Mansini et al., 2014), which means only 39 studies. Table 3 presents the list of papers that used exact methods to solve the portfolio optimization problem, evidencing the strong participation of these methods for the selection of the best assets (stocks).

Most of the studies presented in Table 4, which use heuristics, addressed multi-period, and multi-objective problems. Fuzzy Logic, GA, and Swarm Intelligence Algorithms (emphasis on Particle Swarm Optimization - PSO) are the methods that appear more in the studies.

According to Table 4, among the articles that use heuristics, 23.8\% address the use of hybrid algorithms, $38.09 \%$ work with a single-period, $47.61 \%$ address the multi-period, and $71.4 \%$ work with more than one objective function (Multi-objective). Figure 5 shows the papers' distribution according to the year of publication.

\section{Discussion}

\subsection{Which key methods, tools, or optimization techniques are used in the portfolio optimization problem?}

In 18 studies, the authors use exact techniques to solve the portfolio optimization problem starting from the MV Model, or by adapting it (inserting new parameters instead of the mean and variance) what some authors call the extension of Markowitz model (Ali et al., 2016; Ayub et al., 2015; Babaei et al., 2015; Ban et al., 2016; Bensaïda et al., 2018; Ceren \& Köksalan, 2014; Hu et al., 2015; Kumar \& Mishra, 2017; Levy \& Kaplanski, 2015; Liu \& Zhang, 2015; Marzban et al., 2015; Rezaei Pouya et al., 2016; Rubio et al., 2016; Sharma \& Banerjee, 2015; Le Thi \& Moeini, 2014; Zhang \& Liu, 2014; Zhao et al., 2018b).

Despite the evolution of new techniques to solve portfolio optimization problems (stochastic/heuristic), many investors and researchers prefer exact techniques due to its characteristic of finding the optimal global. Regardless of the degree of difficulty of modeling or computational spending that will be required, exact techniques always return to the optimal solution, so they are so attractive, especially for conservative investors.

The number of published studies that used heuristics to solve the portfolio optimization problem demonstrates the strong participation of these methods (Babaei et al., 2015; Berutich et al., 2016; Chen \& Zhou, 2018; Ertenlice \& Kalayci, 2018; García et al., 2018; Kumar \& Mishra, 2017; Li \& Bao, 2014; Liu \& Zhang, 2015; Macedo et al., 2017; Meghwani \& Thakur, 2018; Mishra et al., 2016; Pai, 2017; Pekár et al., 2016; Qu et al., 2017; Reveiz-Herault, 2016; Rezaei Pouya et al., 2016; Saborido et al., 2016; Silva et al., 2015; Vercher \& Bermúdez, 2015). Only one of the selected studies shows the use of Markov chains (Bensaïda et al., 2018). In Figure 6, the distribution of the resolution techniques for the Portfolio optimization problem is shown. It is possible to notice the strong participation of heuristics solutions, although the exact techniques have an extensive presence.

One of the justifications for the use of metaheuristics is the fact that they find a solution closer to optimal with a lower computational expense than if it were exact methods. Furthermore, depending on the degree of complexity of the model, these techniques converge to the optimal solution faster than the exact ones. Another advantage of heuristics is the ease of working with multi-objective problems and with several restrictions. 


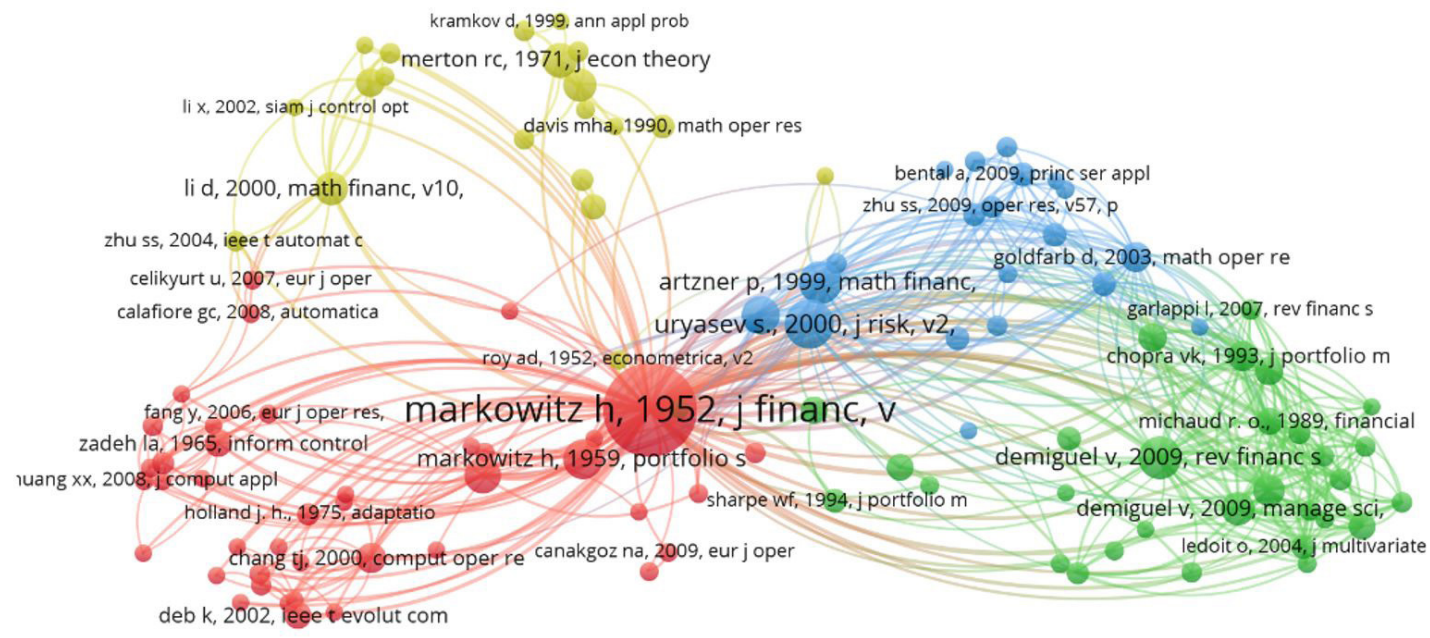

s Vosviewer

Figure 4. Mapping of the most cited authors.

Table 2. Qualification of Journals.

\begin{tabular}{|c|c|c|}
\hline Journal & $\begin{array}{l}\text { Impact factor } \\
\text { (5 years) }\end{array}$ & Papers \\
\hline IEEE TRANSACTIONS ON FUZZY SYSTEMS & 9.340 & 1 \\
\hline MANAGEMENT SCIENCE & 4.927 & 2 \\
\hline SWARM AND EVOLUTIONARY COMPUTATION & 4.607 & 4 \\
\hline APPLIED SOFT COMPUTING (PRINT) & 4.004 & 2 \\
\hline EUROPEAN JOURNAL OF OPERATIONAL RESEARCH & 3.960 & 4 \\
\hline EXPERT SYSTEMS WITH APPLICATIONS & 3.711 & 6 \\
\hline PLOS ONE & 3.352 & 1 \\
\hline OPERATIONS RESEARCH & 3.047 & 1 \\
\hline ELECTRIC POWER SYSTEMS RESEARCH & 2.859 & 1 \\
\hline NEURAL COMPUTING \& APPLICATIONS & 2.697 & 1 \\
\hline INTERNATIONAL JOURNAL OF APPROXIMATE REASONING & 2.504 & 1 \\
\hline JOURNAL OF STATISTICAL MECHANICS-THEORY AND EXPERIMENT & 2.228 & 1 \\
\hline OR-SPEKTRUM & 2.227 & 1 \\
\hline PHYSICA. A (PRINT) & 2.076 & 2 \\
\hline ANNALS OF OPERATION RESEARCH & 1.943 & 2 \\
\hline ECONOMIC MODELLING & 1.844 & 3 \\
\hline INTERNATIONAL JOURNAL OF INFORMATION TECHNOLOGY \& DECISION MAKING & 1.670 & 1 \\
\hline JOURNAL OF THE OPERATIONAL RESEARCH SOCIETY & 1.634 & 1 \\
\hline JOURNAL OF OPTIMIZATION THEORY AND APPLICATIONS & 1.412 & 1 \\
\hline QUANTITATIVE FINANCE (PRINT) & 1.190 & 2 \\
\hline MATHEMATICAL PROBLEMS IN ENGINEERING (PRINT) & 1.156 & 1 \\
\hline CENTRAL EUROPEAN JOURNAL OF OPERATIONS RESEARCH & 0.948 & 1 \\
\hline INTELLIGENT SYSTEMS IN ACCOUNTING, FINANCE \& MANAGEMENT & $\mathrm{N} / \mathrm{D}$ & 1 \\
\hline
\end{tabular}

According to Table 4, about $71 \%$ of articles that address heuristics are about multi-objective portfolio optimization. Multi-objective problems are solved either by a combination that transforms them into the mono objective or taking as an objective function only one objective and turning others into constraints. These are examples of objectives: maximizing return or minimizing measures such as risk, transaction costs, degree of model uncertainty, Value-At-Risk (VaR) and Conditional Value-At-Risk (CVaR). Hybrid algorithms composed of Fuzzy, GA, MOES, and NSGA 11 have been widely used to solve problems with more than one objective function. 
Table 3. Papers that used exact methods.

\begin{tabular}{cc}
\hline References & Technique: Exact \\
\hline Al Janabi (2014) & LVaR \\
Ayub et al. (2015) & DR framework \\
Ackermann et al. (2016) & MV \\
Ali et al. (2016) & MV \\
Algarvio et al. (2017) & PBR \\
Ban et al. (2016) & MedMAD \\
Benati (2015) & Markov chains \\
Bensaïda et al. (2018) & MILP \\
Ceren \& Köksalan (2014) & MinG \\
Ji et al. (2017) & SSDMN \\
Levy \& Kaplanski (2015) & IMPRO \\
Marzban et al. (2015) & MST \\
Ren et al. (2017) & Mean-variance-asymmetry-kurtosis \\
Sharma \& Banerjee (2015) & DCCA \\
Sun \& Liu (2016) & MAD \\
Le Thi \& Moeini (2014) & q-dependent \\
Zhao et al. (2018a) & Temporal network structure \\
Zhao et al. (2018b)
\end{tabular}

MAD: Mean-Absolute Deviation Model; PBR: Performance-based Regularization-Combinatorial optimization; MV: Mean-Variance; MINLP: Mixed-integer nonlinear programming: MedMAD: Median Mean Absolute Deviation (MAD); MILP: Mixed-integer linear programming; 1MPRO: Insured Multi-period Portfolio Robust Optimization linear model; MinG: Minimal Gini model; LVar: Liquidity adjusted value at risk; DR Framework: Extension of the Markowitz model where the proxy variance was substituted for the downside risk as asymmetric lower partial moments (ALPM); SSDMN: Stochastic Domain for mixed normal distributions; DCCA: Method for Cross-Correlation Analysis; q-dependent: q-dependent cross-correlation coefficient; MST: Dynamic portfolio Strategy based on network structures (minimum spanning tree).

Table 4. Papers that used Heuristic methods.

\begin{tabular}{|c|c|c|c|c|c|c|c|}
\hline References & Technique: Exact/Heuristic & Heuristic & $\begin{array}{l}\text { Hybrid } \\
\text { heuristic }\end{array}$ & Single-period & Multi-period & Single-objective & Multi-objective \\
\hline Babaei et al. (2015) & MOPSO & $x$ & & $x$ & & & $x$ \\
\hline Berutich et al. (2016) & GP & $x$ & & & $x$ & & $x$ \\
\hline Chen \& Zhou (2018) & MOPSO & $x$ & & & $x$ & & $x$ \\
\hline García et al. (2018) & $\mathrm{GA}$ and $\mathrm{TS}$ & $x$ & & & $x$ & $x$ & \\
\hline Kumar \& Mishra (2017) & $\mathrm{M}-\mathrm{CABC}$ & $x$ & & $x$ & & & $x$ \\
\hline Li \& Bao (2014) & Immunological algorithm & $x$ & & $x$ & & & $x$ \\
\hline Liu \& Zhang (2015) & GA with Fuzzy & & $x$ & & $x$ & & $x$ \\
\hline Macedo et al. (2017) & NSGA 11 and SPEA 2 & $x$ & & & $x$ & & $x$ \\
\hline $\begin{array}{c}\text { Meghwani \& Thakur } \\
\text { (2018) }\end{array}$ & NSGA 11; MOEA/D and GWASF-GA & $x$ & & & $x$ & & $\mathrm{x}$ \\
\hline Mishra et al. (2016) & SR-MOPSO & $x$ & & $x$ & & & $x$ \\
\hline Pai (2017) & MODE/Fuzzy and MOES/Fuzzy) & & $x$ & & $x$ & & $x$ \\
\hline Pekár et al. (2016) & Algorithm of differential evolution & $x$ & & $x$ & & $x$ & \\
\hline Qu et al. (2017) & (NMOEA/D) & $x$ & & & $x$ & & $x$ \\
\hline Reveiz-Herault (2016) & $\mathrm{GA}$ & $x$ & & N/D & $\mathrm{N} / \mathrm{D}$ & $x$ & \\
\hline $\begin{array}{c}\text { Rezaei Pouya et al. } \\
\text { (2016) }\end{array}$ & IWO and PSO & $x$ & & $x$ & & $\mathrm{x}$ & \\
\hline Rubio et al. (2016) & FTS & $x$ & & & & & \\
\hline Rubio, et al. (2017) & FLR & $x$ & & & & & \\
\hline Saborido et al. (2016) & MDRS & & $x$ & $x$ & & & $\mathrm{x}$ \\
\hline Silva et al. (2015) & GA & $x$ & & & $x$ & & $x$ \\
\hline $\begin{array}{l}\text { Vercher \& Bermúdez } \\
\text { (2015) }\end{array}$ & GA with Fuzzy & & $x$ & $\mathrm{x}$ & & & $\mathrm{x}$ \\
\hline Zhang \& Liu (2014) & GA with Fuzzy & & $x$ & & $x$ & & $x$ \\
\hline
\end{tabular}

GA: Genetic algorithm; Fuzzy: Fuzzy Programming; MOPSO: multi-objective particle swarm optimization; TS: Tabu Search; NSGA 11: Non-dominated sorting genetic algorithm 11; SPEA 2: strength Pareto evolutionary algorithm 2; GP: Genetic Programming; NMOEA/D: Normalized Multi-objective Evolutionary Algorithm based on Decomposition; MODE/Fuzzy: Multi-objective differential Evolution/Fuzzy; MOES/Fuzzy: Multi-Objective Evolution Strategy/Fuzzy; GWASF-GA: Global Weighting Achievement Scalarizing Function Genetic Algorithm; M-CABC: Artificial Bee Colony Algorithm based on Multi-objective covariance; SR-MOPSO: Self-regulating multi-objective particle swarm optimization; IWO: Invasive Weed Optimization; PSO: Particle Swarm Optimization; FTS: Methods of weighted diffuse time series: FLR: Fuzzy Logic Relation; MDRS: Mean Downside Risk-Skewness; MOEA/D: Multi-objective evolutionary algorithm based on decomposition; N/D: Not Defined. 


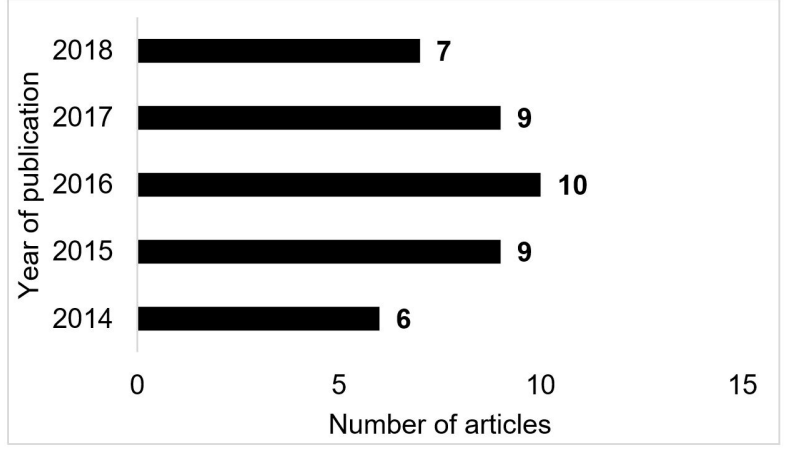

Figure 5. Papers distribution per year of publication.

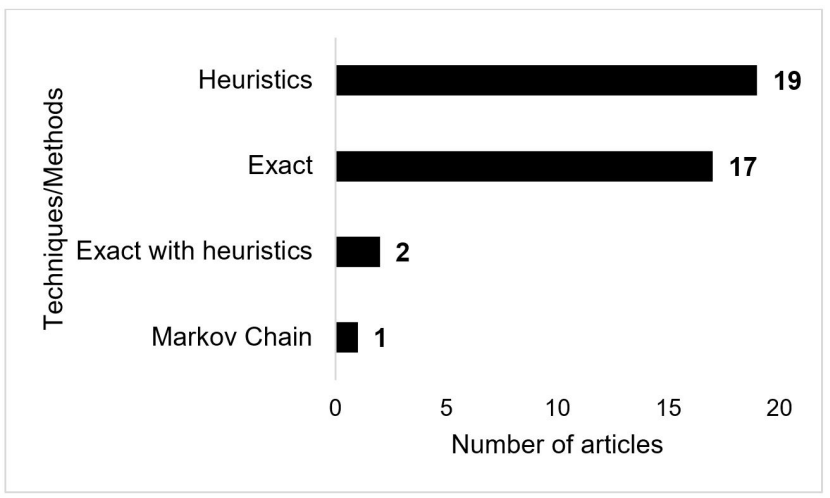

Figure 6. Papers distribution per techniques/methods for portfolio optimization.

The main goal of hybridization is to unite the advantages of two or more each algorithm and to build a robust model. For example, Zhang \& Liu (2014), propose a hybrid model using Fuzzy and Genetic Algorithm (GA). With Fuzzy, it is possible to solve Multi-objective problems by transforming the problem single-objective. In addition, expert knowledge can be inserted in the Portfolio Selection Model (optimization) using Fuzzy (Liu \& Zhang, 2015; Rubio et al., 2016; Zhang \& Liu, 2014).

Two techniques draw attention to the development of new studies: ABC and GP. The ABC, when compared to other populations and stochastic algorithms of the same class, presents similar or better performance using fewer control parameters than other algorithms (Ertenlice \& Kalayci, 2018; Kumar \& Mishra, 2017). About GP, the great advantage of this model is the fact that it allows the management of a stock portfolio in an automatic way, without requiring the help of financial market specialists, without having to make constant adjustments in the model (Berutich et al., 2016).

The choice of method to solve the optimization problem depends primarily on what the researcher wants to obtain concerning the expected results:

a) The complexity of the model, because the difficult model is commonly solved by heuristics;

b) The time spent to get a solution (more powerful methods like the exact ones spend more time and deliver optimal solutions, and heuristic methods spend less time but deliver an approximate solution that can be considered feasible).

Furthermore, to these approaches, the investor can choose the backtesting method, where he can verify the efficiency of the entire strategy in the most varied combinations between mathematical models, exact and heuristic methods, restrictions, and strategy $1 / \mathrm{N}$ by DeMiguel et al. (2009a). Generally, the decision-making between using $1 / \mathrm{N}$ in the opposite of optimization is based on metrics used to assess the degree of uncertainty of the model, and if this measure is high, the $1 / \mathrm{N}$ strategy is preferred.

DeMiguel et al. (2009a) report that the portfolio optimization strategy has advantages over Benchmark 1/N in three situations: (1) if there is an abundance of historical data available; (2) if the Sharpe ex ant value (expected) of the efficient medium variance portfolio is substantially higher than the Sharpe value of the 
$1 / \mathrm{N}$ portfolio; and (3) the number of assets that will make up the portfolio is small. The first two conditions are intuitive, and the reason for the latter condition is that a smaller number of assets implies fewer parameters to be estimated and, therefore, less space for estimation error, and this makes $1 / \mathrm{N}$ diversification less effective than diversification by optimization.

\subsection{Which realistic constraints are used?}

Constraints make the model more complex to be solved; however, they make the portfolio optimization process much easier. Table 5 shows the constraints, the authors who cited them, and the method used by the authors to solve the problems. The non-negativity constraints and the total value available must be fully invested, have not entered the count, because they are considered trivial in portfolio optimization models. There is an extension to comment on the databases that the authors used to test their algorithms in this section. In some studies, the OR-Library instance was used that allows free access to the data. The data sources are well diversified, and among the papers, only one used data from Brazil through the Thomson-Reuters Datastream platform (Macedo et al., 2017).

With several studies that succeeded in the model proposed by Markowitz (1952), some constraints were proposed, among them the cardinality constraints and minimum purchase limits constraints. These constraints provides to the model a non-convex search space, which makes the problem difficult (in terms of computational cost and response time) to be solved using exact approaches (Ertenlice \& Kalayci, 2018; Zhang et al., 2018; Berutich et al., 2016; García et al., 2018; Kumar \& Mishra, 2017; Meghwani \& Thakur, 2018; Pai, 2017; Qu et al., 2017; Rezaei Pouya et al., 2016). Below there is the description of the meaning of each constraint of Table 5:

Table 5. Realistic constraints.

\begin{tabular}{|c|c|c|c|}
\hline Author(s) & Heuristic method & Realistic constraints & Database \\
\hline Babaei et al. (2015) & MOPSO & threshold constraints; Cardinality constraints; & S\&P500 \\
\hline Berutich et al. (2016) & GP & $\mathrm{N} / \mathrm{D}$ & $\begin{array}{l}\text { Stock Market Espanha } \\
\text { (índice 1BEX35) }\end{array}$ \\
\hline Chen \& Zhou (2018) & MOPSO, NSGA 11 and SPEA 2 & Long-only constraint & Stock Market China \\
\hline García et al. (2018) & GA and TS & Cardinality constraints & OR-Library \\
\hline Kumar \& Mishra (2017) & $\mathrm{M}-\mathrm{CABC}$ & $\begin{array}{l}\text { Cardinality constraints; threshold constraints; } \\
\text { Long only constraint; }\end{array}$ & OR-Library \\
\hline Li \& Bao (2014) & Immunological algorithm & $\begin{array}{l}\text { Cardinality constraints; threshold constraints; } \\
\text { Transaction cost; }\end{array}$ & $\begin{array}{l}\text { Indices: Hang Seng 33, DAX } \\
\text { 200, FTSE 100, S \& P } 100 \text { e } \\
\text { Nikkei } 225 .\end{array}$ \\
\hline Liu \& Zhang (2015) & GA with Fuzzy & $\begin{array}{c}\text { Cardinality constraints; Transaction cost; } \\
\text { Transaction lots; }\end{array}$ & Stock market China \\
\hline Macedo et al. (2017) & NSGA 11 and SPEA 2 & $\begin{array}{c}\text { Cardinality constraints; Transaction cost; } \\
\text { Transaction lots; }\end{array}$ & $\begin{array}{l}\text { Thompson-Reuters } \\
\text { Datastream (agora Eikon) }\end{array}$ \\
\hline Meghwani \& Thakur (2018) & NSGA 11, MOEA/D, and GWASF-GA & $\begin{array}{l}\text { Cardinality constraints; Quantity constraint; } \\
\text { self-financing constraint; Transaction cost. }\end{array}$ & $\begin{array}{l}\text { Fama and French Data } \\
\text { Library }\end{array}$ \\
\hline Mishra et al. (2016) & SR-MOPSO & Cardinality constraints; threshold constraints; & OR-Library \\
\hline Pai (2017) & MODE/Fuzzy and MOES/Fuzzy & Long-only constraint & $\begin{array}{l}\text { Índice BSE (Bombaim Stock } \\
\text { Exchange, Índia) }\end{array}$ \\
\hline Pekár et al. (2016) & Algorithm of differential evolution & $\begin{array}{l}\text { Non-constrained problem with continuous } \\
\text { variables }\end{array}$ & Indice Dow Jones \\
\hline Rezaei Pouya et al. (2016) & IWO and PSO & $\begin{array}{l}\text { Sector Capitalization; Transaction lots; } \\
\text { Cardinality constraints; threshold constraints; }\end{array}$ & $\begin{array}{l}\text { Tehran Stock Exchange } \\
\text { Market in } 2013\end{array}$ \\
\hline Qu et al. (2017) & NMOEA/D & $\mathrm{N} / \mathrm{D}$ & Stock Market China \\
\hline Reveiz-Herault (2016) & $\mathrm{GA}$ & Tracking Error Constraints. & Índice de títulos dos EUA \\
\hline Saborido et al. (2016) & MDRS & Cardinality constraints & $\begin{array}{l}\text { Stock Market Espanha } \\
\quad \text { (indice IBEX35) }\end{array}$ \\
\hline Silva et al. (2015) & $\mathrm{GA}$ & $\begin{array}{l}\text { Cardinality constraint; Quantity constraint; } \\
\text { Long only constraint; Transaction costs. }\end{array}$ & S\&P500 \\
\hline Vercher \& Bermúdez (2015) & GA with Fuzzy & Cardinality Constraints & $\begin{array}{l}\text { Stock Market Espanha } \\
\quad \text { (indice 1BEX35) }\end{array}$ \\
\hline Zhang \& Liu (2014) & GA with Fuzzy & $\begin{array}{l}\text { self-financing constraint; the Long only } \\
\text { constraint }\end{array}$ & Stock market Shangai \\
\hline
\end{tabular}

GA: Genetic algorithm; Fuzzy: Fuzzy Programming; MOPSO: multi-objective particle swarm optimization; TS: Tabu Search; NSGA 11: Non-dominated sorting genetic algorithm 11; SPEA 2: strength Pareto evolutionary algorithm 2; GP: Genetic Programming; NMOEA/D: Normalized Multi-objective Evolutionary Algorithm based on Decomposition; MODE/Fuzzy: Multi-objective differential Evolution/Fuzzy; MOES/Fuzzy: Multi-Objective Evolution Strategy/Fuzzy; GWASF-GA: Global Weighting Achievement Scalarizing Function Genetic Algorithm; M-CABC: Artificial Bee Colony Algorithm based on Multi-objective covariance; SR-MOPSO: Self-regulating multi-objective particle swarm optimization; IWO: Invasive Weed Optimization; PSO: Particle Swarm Optimization; MDRS: Mean Downside Risk-Skewness; MOEA/D: Multi-objective evolutionary algorithm based on decomposition; N/D: Not Defined. 
a) Transaction costs: in the financial markets, there are transaction costs arising from the process of purchasing and selling bonds and investment portfolio review;

b) Transaction lots - rounds: is a minimum transaction unit required to invest in an asset;

c) Cardinality constraint: restricts the number of bonds that will compose the investment portfolio;

d) Investment threshold constraints: these constraints define upper limits of the ratio-amount of each asset held in the portfolio;

e) Decision dependency constraints: decision dependency requirements that are common in financial transactions;

f) Quantity constraint: the quantity constraint is used to limit the position size by placing maximum and minimum values. The minimum limit is set to $5 \%$ of the portfolio value at the time of the transaction, and the maximum value is $20 \%$. The lower limit avoids virtually insignificant positions for the portfolio performance, and the upper limit averts too much exposure or weight for any stock;

g) Long-only constraint: means that the realization of selling short operations is not permitted, i.e., the weight invested in any stock is always positive;

h) Self-financing constraint: no money is withdrawn or added during the operation.

\subsection{What type of analysis is done regarding the stock: fundamental, technical, or mixed (fundamental and technical)?}

There are few studies using fundamental analysis (Ackermann et al., 2016; Algarvio et al., 2017; Liu \& Zhang, 2015; Reveiz-Herault, 2016), and one of the rationales is the complexity in analyzing the companies' financial performance data (investors who use fundamental analysis are more conservative and invest in long term). The technical analysis for being simpler, since it analyzes few variables, has an advantage regarding the fundamental type in terms of the analysis speed in daily operations, for example.

Most papers use technical analysis (Al Janabi, 2014; Ali et al., 2016; Ban et al., 2016; Benati, 2015; Bensaïda et al., 2018; Ceren \& Köksalan, 2014; Kumar \& Mishra, 2017; Levy \& Kaplanski, 2015; Li \& Bao, 2014; Macedo et al., 2017; Marzban et al., 2015; Mishra et al., 2016; Qu et al., 2017; Rubio et al., 2016; Sharma \& Banerjee, 2015; Sun \& Liu, 2016; Le Thi \& Moeini, 2014; Zhang \& Liu, 2014), because this is easy to access in instances and/or stockbrokers. In addition, investors who use this data want to get rapid gains in daily, weekly, or monthly operations, identifying through simpler analyses and fewer variables, the opportunities offered by the market. In Figure 7 presents the distribution of the types of analysis in the selected papers.

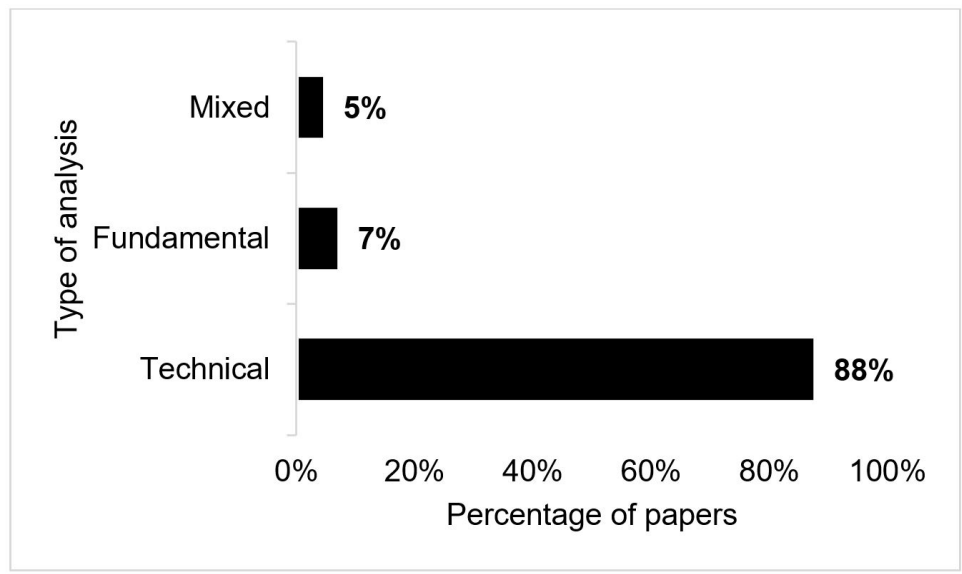

Figure 7. Papers distribution per type of analysis. 
Silva et al. (2015), propose a mixed approach of the two types of analysis, and demonstrate through simulations that the stock optimization based on financial indices (arising from fundamental analysis) can be used to choose the best companies in operational terms, obtaining returns above market average with low variations in their returns. In the points below, the financial indices that enable the company's fundamental analysis in terms of profitability, liquidity, debt, and growth are mentioned (Silva et al., 2015):

a) Debt Index (DR): is used to measure the company's indebtedness level;

b) Net Return (ROE): measures financial performance in the case of generating profits using company assets;

c) Profit Margin Index (PM): assess the company profitability by calculating the percentage of retained earnings after paying the operational, administrative and financial costs, in addition to taxes;

d) Price-Profit Index (PER): is a valuation ratio of the current price of a company's share compared to its earnings per share, and is used to choose companies that are less valued in the market;

e) Revenue growth percentage (GR): is an economic indicator that shows the business evolution;

f) Positive Trend of net profit (NI): shows the increase in profits, and aims to show the companies with the highest growth;

g) Common Shares in circulation (CSO): represents the fundamental owner of the corporation by shareholders. When the company issues share, this number is added to the previous value in the balance sheet, representing an increase in the total number of shares and distribution of the company value by a larger number of shares.

The following paragraphs are the parameters for a technical negotiation used in (Silva et al., 2015):

a) The global value of each stock: is a daily assessment of the quarterly financial indexes and the daily PER, which is updated using the closing price to classify the algorithm actions to make a business decision;

b) Stop loss and protection of capital: are predetermined policies that reduce the portfolio exposure, leaving a losing position, not allowing one or more investments to continue to lose money and protect the remaining capital available to continue the investment activity;

c) Take profit: percentage of pre-established profit, and when it is reached the stock is sold;

d) Position Size: is the percentage of the current value of the portfolio to invest in each new stock. This defines the level of concentration or diversification in the portfolio.

\subsection{Which software/programming language is used?}

Among the explored studies, 26, did not inform the software or programming language that they used to implement the proposed model, but only the pseudocodes or the mathematical model. The ratio distribution of software or programming language is given by Figure 8. There are tools with all kinds of features: free, paid easy learning and that have libraries/packages (which can be used by researchers who want to solve a problem without necessarily learning a programming language).

$\mathrm{R}$ software offers some advantages such as the best learning curve, a wide variety of packages ready for optimization or Data Science, and active forums. Despite requiring certain programming knowledge, $\mathrm{R}$ appears as a nice option for portfolio optimization scholars for allowing a greater learning curve than other languages.

According to Karakalidis \& Sifaleras (2017), AMPL is an easy-to-learn language where the researcher implements the mathematical model simply using a natural language and notations very close to those used in the writing of the model, and allows the call optimization software. Among these solvers, we can highlight CPLEX $^{\circledR}$, GUROBI Optimizer ${ }^{\circledR}$, and KNITRO ${ }^{\circledR}$ (used for nonlinear problems). The choice of solver depends on the problem to be solved (linear programming, quadratic programming, mixed entire quadratic, nonlinear problems). The solvers are available for free in the student version, however, with limitations, since, depending on the size/complexity of the problem, it is necessary to acquire more complete versions that are paid for. 


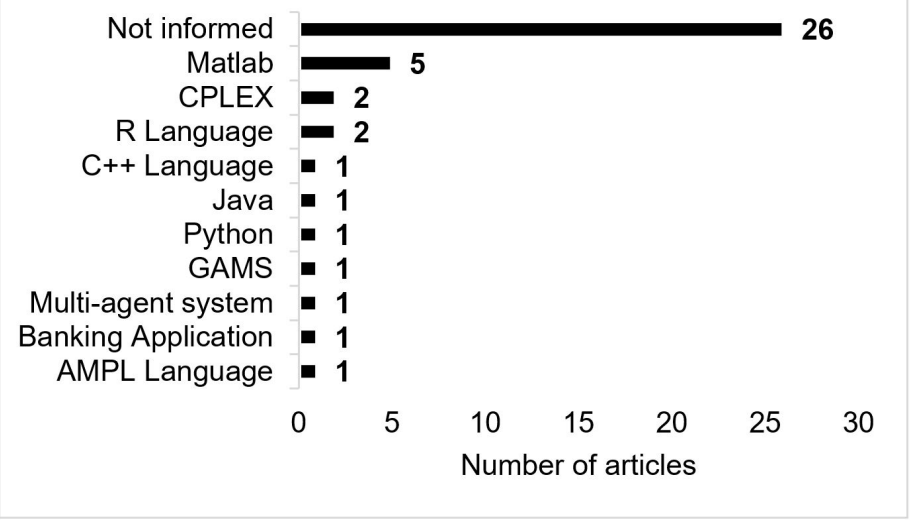

Figure 8. Papers distribution per software/programming language.

The Python language has interesting features that enable the construction of a flexible program or algorithm. In addition, object-oriented programming, materials available on the Internet, and calculation speed are other advantages of this programming language (Berutich et al., 2016; Goodman \& Brette, 2008; McKinney, 2010; Mitchell et al., 2011). In addition to the libraries, there is a module called Scikit-Learn, that integrates a wide range of state-of-the-art machine learning algorithms for supervised and unsupervised mid-scale issues. This package focuses on taking machine learning for non-specialists using a high-level, general-purpose language (Pedregosa et al., 2011).

Despite some differences, Python represents, in this discussion, the category of languages composed of R, $\mathrm{C}_{++}$, and Java, as all require certain knowledge about programming regardless of having libraries/packages that facilitate the user's life, and are available for free. Additionally, due to flexibility, these languages are better for researchers or investors who want to work with heuristics to solve optimization problems.

\section{Conclusions}

To provide a comprehensive systematic review, with the classification of listed articles and suggestions for future research, we reviewed 41 articles published in scientific journals that address topics related to portfolio optimization: exact and heuristic methods, realistic restrictions, markets evaluated (benchmark), stock analysis types, and programming software/languages used. Although the research has not been exhaustive, it is believed that this review can facilitate the accumulation of knowledge and further studies in this area of research. The implications of this research are shown in the following paragraphs.

Regardless of the method used, the use of techniques to make estimates of future returns is a trend in studies on portfolio optimization, since the error in estimating parameters of the model compromises the quality of optimization. Techniques such as robust optimization, Bayesian statistics, Neural Networks and Fuzzy logic are used to amortize the estimation error.

Themes such as robust optimization, dynamic optimization, multi-objective problems, and hybrid algorithms often appear in portfolio optimization jobs. In addition to showing how diverse methods to solve the Portfolio Optimization problem are, these approaches offer a range of possibilities for combinations with each other, which can be used to increase their ability to address the problem of portfolio optimization.

The databases used to test the algorithms/tools are important for the functional analyses of the methods used. Data to test the optimization model or algorithm can be obtained on paid platforms such as Thomson Reuters or instances such as OR-Library and Fame-French that make the data available for free.

About programming language, there is the possibility of using ready-made optimization software or programming using programming languages. AMPL is a good option for the researcher who does not want to deepen his knowledge about programming and prefers to use optimization software to solve problems. Languages such as Python, $\mathrm{R}, \mathrm{C}_{++}$, and Java require certain knowledge about programming from the user, but in return, they offer advantages such as calculation speed, flexibility in model building, active communities/ forums, and free availability for users. 
The gaps identified in the literature and the suggestions for future research on the theme portfolio optimization are as follows: Develop robust predictors for the risk-return binomial, aiming to increase the capacity of the model to optimize an investment portfolio; Studying the addition of constraints based on fundamental analysis of shares, that is, within this type of analysis what would be the constraints that, when added, make the mathematical model more efficient for portfolio optimization; Which constraint could be added to prevent the model from seeking shares highly influenced by market volatility (and consequently by the emotions of those people who invest); The computational complexity of the Portfolio Optimization Problem, along with the practical importance of portfolio management, triggers researchers' motivation to design efficient algorithms. Therefore, improvements related to computational efficiency (parameter calibration, hybridization, among others) to deal with methodologies of algorithms, heuristic and accurate, are always welcome.

Although the filters and search strategies have been defined to choose the best and most current studies on the topic under discussion, it is believed that at some point, these strategies/filters may have prevented finding more relevant studies. The non-English publications filter may have deleted relevant works written in other languages. However, we are convinced that we have compiled the main work to support the discussion proposed in the Systematic Review Protocol (Table 1).

\section{Acknowledgements}

To the Coordination for the Improvement of Higher Education Personnel (CAPES) for subsidizing this research. The authors declare that they have no conflict of interest.

\section{References}

Ackermann, F., Pohl, W., \& Schmedders, K. (2016). Optimal and naive diversification in currency markets. Management Science, 63(10), 3347-3360. http://dx.doi.org/10.1287/mnsc.2016.2497.

Al Janabi, M. A. M. (2014). Optimal and investable portfolios: an empirical analysis with scenario optimization algorithms under crisis market prospects. Economic Modelling, 40, 369-381. http://dx.doi.org/10.1016/j.econmod.2013.11.021.

Algarvio, H., Lopes, F., Sousa, J., \& Lagarto, J. (2017). Multi-agent electricity markets: retailer portfolio optimization using Markowitz theory. Electric Power Systems Research, 148, 282-294. http://dx.doi.org/10.1016/j.epsr.2017.02.031.

Ali, Ö. G., Akçay, Y., Sayman, S., Yılmaz, E., \& Özçelik, M. H. (2016). Cross-selling investment products with a Win-win perspective in portfolio optimization. Operations Research, 65(1), 55-74. http://dx.doi.org/10.1287/opre.2016.1556.

Ayub, U., Shah, S. Z. A., \& Abbas, Q. (2015). Robust analysis for downside risk in portfolio management for a volatile stock market. Economic Modelling, 44, 86-96. http://dx.doi.org/10.1016/j.econmod.2014.10.001.

Babaei, S., Sepehri, M. M., \& Babaei, E. (2015). Multi-objective portfolio optimization considering the dependence structure of asset returns. European Journal of Operational Research, 244(2), 525-539. http://dx.doi.org/10.1016/j.ejor.2015.01.025.

Ban, G.-Y., El Karoui, N., \& Lim, A. E. B. (2016). Machine learning and portfolio optimization. Management Science, 64(3), 1136-1154. http://dx.doi.org/10.1287/mnsc.2016.2644.

Bastos, L. D. S. L., Mendes, M. L., Nunes, D. R. D. L., Melo, A. C. S., \& Carneiro, M. P. (2017). A systematic literature review on the joint replenishment problem solutions: 2006-2015. Production, 270), 27. http://dx.doi.org/10.1590/0103-6513.222916.

Behr, P., Guettler, A., \& Miebs, F. (2013). On portfolio optimization: imposing the right constraints. Journal of Banking \& Finance, 37(4), 1232-1242. http://dx.doi.org/10.1016/j.jbankfin.2012.11.020.

Benati, S. (2015). Using medians in portfolio optimization. The Journal of the Operational Research Society, 66(5), 720-731. http:// dx.doi.org/10.1057/jors.2014.57.

Bensaïda, A., Boubaker, S., \& Nguyen, D. K. (2018). The shifting dependence dynamics between the G7 stock markets. Quantitative Finance, 18(5), 801-812. http://dx.doi.org/10.1080/14697688.2017.1419628.

Berutich, J. M., López, F., Luna, F., \& Quintana, D. (2016). Robust technical trading strategies using GP for algorithmic portfolio selection. Expert Systems with Applications, 46, 307-315. http://dx.doi.org/10.1016/j.eswa.2015.10.040.

Brodie, J., Daubechies, 1., De Mol, C., Giannone, D., \& Loris, 1. (2009). Sparse and stable Markowitz portfolios. Proceedings of the National Academy of Sciences of the United States of America, 106(30), 12267-12272. http://dx.doi.org/10.1073/pnas.0904287106. PMid:19617537.

Ceren, T. Ş., \& Köksalan, M. (2014). Effects of Multiple Criteria on Portfolio Optimization. International Journal of Information Technology \& Decision Making, 13(01), 77-99. http://dx.doi.org/10.1142/S0219622014500047.

Chen, C., \& Zhou, Y. (2018). Robust multiobjective portfolio with higher moments. Expert Systems with Applications, 100, $165-181$. http://dx.doi.org/10.1016/j.eswa.2018.02.004.

Dai, Z., \& Wen, F. (2018). Some improved sparse and stable portfolio optimization problems. Finance Research Letters, 27, 46-52. http://dx.doi.org/10.1016/j.frl.2018.02.026.

DeMiguel, V., Garlappi, L., \& Uppal, R. (2009a). Optimal versus naive diversification: How inefficient is the 1/N portfolio strategy? Review of Financial Studies, 22(5), 1915-1953. http://dx.doi.org/10.1093/rfs/hhm075.

DeMiguel, V., Garlappi, L., Nogales, F. J., \& Uppal, R. (2009b). A generalized approach to portfolio optimization: improving performance by constraining portfolio norms. Management Science, 55(5), 798-812. http://dx.doi.org/10.1287/mnsc.1080.0986. 
Ertenlice, 0., \& Kalayci, C. B. (2018). A survey of swarm intelligence for portfolio optimization: algorithms and applications. Swarm and Evolutionary Computation, 39, 36-52. http://dx.doi.org/10.1016/j.swevo.2018.01.009.

Fabozzi, F. J., Kolm, P. N., Pachamanova, D. A., \& Focardi, S. M. (2007). Robust portfolio optimization. Journal of Portfolio Management, 33(3), 40-48. http://dx.doi.org/10.3905/jpm.2007.684751.

Fan, J., Zhang, J., \& Yu, K. (2012). Vast portfolio selection with gross-exposure constraints. Journal of the American Statistical Association, 107498), 592-606. http://dx.doi.org/10.1080/01621459.2012.682825. PMid:23293404.

Furlan, P. K., \& Laurindo, F. J. B. (2017). Agrupamentos epistemológicos de artigos publicados sobre big data analytics. Transinformação, 29(1), 91-100. http://dx.doi.org/10.1590/2318-08892017000100009.

García, F., Guijarro, F., \& Oliver, J. (2018). Index tracking optimization with cardinality constraint: a performance comparison of genetic algorithms and tabu search heuristics. Neural Computing \& Applications, 30(8), 2625-2641. http://dx.doi.org/10.1007/ s00521-017-2882-2.

Goodman, D., \& Brette, R. (2008). Brian: a simulator for spiking neural networks in Python. Frontiers in Neuroinformatics, 2, 5. http:// dx.doi.org/10.3389/neuro.11.005.2008. PMid:19115011.

Hu, Y., Liu, K., Zhang, X., Su, L., Ngai, E. W. T., \& Liu, M. (2015). Application of evolutionary computation for rule discovery in stock algorithmic trading: a literature review. Applied Soft Computing, 36, 534-551. http://dx.doi.org/10.1016/j.asoc.2015.07.008.

Ji, R., Lejeune, M. A., \& Prasad, S. Y. (2017). Properties, formulations, and algorithms for portfolio optimization using Mean-Gini criteria. Annals of Operations Research, 248(1-2), 305-343. http://dx.doi.org/10.1007/s10479-016-2230-4.

Karakalidis, A., \& Sifaleras, A. (2017). Solving portfolio optimization problems using AMPL. In: E. Grigoroudis \& M. Doumpos (Eds.), Operational research in business and economics (pp. 167-184). Cham: Springer. http://dx.doi.org/10.1007/978-3-319-33003-7_8.

Kumar, D., \& Mishra, K. K. (2017). Portfolio optimization using novel co-variance guided Artificial Bee Colony algorithm. Swarm and Evolutionary Computation, 34(2), 353-369. http://dx.doi.org/10.1016/j.swevo.2016.11.003.

Le Thi, H. A., \& Moeini, M. (2014). Long-short portfolio optimization under cardinality constraints by difference of convex functions algorithm. Journal of Optimization Theory and Applications, 161(1), 199-224. http://dx.doi.org/10.1007/s10957-012-0197-0.

Levy, M., \& Kaplanski, G. (2015). Portfolio selection in a two-regime world. European Journal of Operational Research, $242(2), 514-524$. http://dx.doi.org/10.1016/j.ejor.2014.10.012.

Li, Q., \& Bao, L. (2014). Enhanced index tracking with multiple time-scale analysis. Economic Modelling, 39, 282-292. http://dx.doi. org/10.1016/j.econmod.2014.03.009.

Liu, Y.-J., \& Zhang, W.-G. (2015). A multi-period fuzzy portfolio optimization model with minimum transaction lots. European Journal of Operational Research, 10(2), 143-164. http://dx.doi.org/10.1016/j.ejor.2014.10.061.

Macedo, L. L., Godinho, P., \& Alves, M. J. (2017). Mean-semivariance portfolio optimization with multiobjective evolutionary algorithms and technical analysis rules. Expert Systems with Applications, 79, 33-43. http://dx.doi.org/10.1016/j.eswa.2017.02.033.

Mansini, R., Ogryczak, W., \& Speranza, M. G. (2014). Twenty years of linear programming based portfolio optimization. European Journal of Operational Research, 234(2), 518-535. http://dx.doi.org/10.1016/j.ejor.2013.08.035.

Markowitz, H. (1952). Portfolio selection. The Journal of Finance, 71), 77-91. http://dx.doi.org/10.1111/j.1540-6261.1952.tb01525.x.

Marzban, S., Mahootchi, M., \& Arshadi Khamseh, A. (2015). Developing a multi-period robust optimization model considering American style options. Annals of Operations Research, 233(1), 305-320. http://dx.doi.org/10.1007/s10479-013-1461-x.

McKinney, W. (2010). Data Structures for Statistical Computing in Python. Proceedings of the 9th Python in Science Conference, 1697900(Scipy), 50-59. Retrieved in 2019, November 27, from http://conference.scipy.org/proceedings/scipy2010/pdfs/proceedings.pdf

Meghwani, S. S., \& Thakur, M. (2018). Multi-objective heuristic algorithms for practical portfolio optimization and rebalancing with transaction cost. Applied Soft Computing, 67, 865-894. http://dx.doi.org/10.1016/j.asoc.2017.09.025.

Merton, R. C. (1971). Optimum consumption and portfolio rules in a continuous-time model. In Stochastic optimization models in finance (pp. 621-661). New York: Academic Press.

Mishra, S. K., Panda, G., \& Majhi, B. (2016). Prediction based mean-variance model for constrained portfolio assets selection using multiobjective evolutionary algorithms. Swarm and Evolutionary Computation, 28, 117-130. http://dx.doi.org/10.1016/j.swevo.2016.01.007.

Mitchell, S., O'Sullivan, M., \& Dunning, 1. (2011). PuLP: a linear programming toolkit for Python. Auckland: The University of Auckland. Retrieved in 2019, November 27, from http://www.optimization-online.org/DB_FlLE/2011/09/3178.pdf

Morandi, M. l. W. M., \& Camargo, L. F. R. (2015). Revisão sistemática da literatura. In A. Dresch, D. P. Lacerda \& J. A. V. Antunes Jr. (Eds.), (pp. 141-175). Design science research: método de pesquisa para avanço da ciência e tecnologia. Porto Alegre: The Bookman.

Pai, G. A. V. (2017). Fuzzy decision theory based metaheuristic portfolio optimization and active rebalancing using interval type-2 fuzzy sets. IEEE Transactions on Fuzzy Systems, 25(2), 377-391. http://dx.doi.org/10.1109/TFUZZ.2016.2633972.

Pedregosa, F., Varoquaux, G., Gramfort, A., Michel, V., Thirion, B., Grisel, O., Blondel, M., Prettenhofer, P., Weiss, R., Dubourg, V., Vanderplas, J., Passos, A., Cournapeau, D., Brucher, M., Perrot, M., \& Duchesnay, É. (2011). Scikit-learn: machine learning in Python. Journal of Machine Learning Research, 12(1), 2825-2830.

Pekár, J., Čičková, Z., \& Brezina, l. (2016). Portfolio performance measurement using differential evolution. Central European Journal of Operations Research, 24(2), 421-433. http://dx.doi.org/10.1007/s10100-015-0393-8.

Pflug, G. C., Pichler, A., \& Wozabal, D. (2012). The 1/N investment strategy is optimal under high model ambiguity. Journal of Banking \& Finance, 36(2), 410-417. http://dx.doi.org/10.1016/j.jbankfin.2011.07.018.

Pflug, G., \& Wozabal, D. (2007). Ambiguity in portfolio selection. Quantitative Finance, 7(4), 435-442. http://dx.doi. org/10.1080/14697680701455410.

Qu, B. Y., Zhou, Q., Xiao, J. M., Liang, J. J., \& Suganthan, P. N. (2017). Large-scale portfolio optimization using multiobjective evolutionary algorithms and preselection methods. Mathematical Problems in Engineering, 2017, 1-14. http://dx.doi.org/10.1155/2017/4197914

Ren, F., Lu, Y. N., Li, S. P., Jiang, X. F., Zhong, L. X., \& Qiu, T. (2017). Dynamic portfolio strategy using clustering approach. PLoS One, 12(1), e0169299. http://dx.doi.org/10.1371/journal.pone.0169299. PMid:28129333. 
Reveiz-Herault, A. (2016). An active asset management investment process for drawdown-averse investors. Intelligent Systems in Accounting, Finance \& Management, 23(1-2), 85-96. http://dx.doi.org/10.1002/isaf.1375.

Rezaei Pouya, A., Solimanpur, M., \& Jahangoshai Rezaee, M. (2016). Solving multi-objective portfolio optimization problem using invasive weed optimization. Swarm and Evolutionary Computation, 28, 42-57. http://dx.doi.org/10.1016/j.swevo.2016.01.001.

Rubio, A., Bermúdez, J. D., \& Vercher, E. (2016). Forecasting portfolio returns using weighted fuzzy time series methods. International Journal of Approximate Reasoning, 75, 1-12. http://dx.doi.org/10.1016/j.jar.2016.03.007.

Rubio, A., Bermúdez, J. D., \& Vercher, E. (2017). Improving stock index forecasts by using a new weighted fuzzy-trend time series method. Expert Systems with Applications, 76, 12-20. http://dx.doi.org/10.1016/j.eswa.2017.01.049.

Saborido, R., Ruiz, A. B., Bermúdez, J. D., Vercher, E., \& Luque, M. (2016). Evolutionary multi-objective optimization algorithms for fuzzy portfolio selection. Applied Soft Computing, 39, 48-63. http://dx.doi.org/10.1016/j.asoc.2015.11.005.

Sharma, C., \& Banerjee, K. (2015). A study of correlations in the stock market. Physica A, 432, 321-330. http://dx.doi.org/10.1016/j. physa.2015.03.061.

Silva, A., Neves, R., \& Horta, N. (2015). A hybrid approach to portfolio composition based on fundamental and technical indicators. Expert Systems with Applications, 42(4), 2036-2048. http://dx.doi.org/10.1016/j.eswa.2014.09.050.

Sun, X., \& Liu, Z. (2016). Optimal portfolio strategy with cross-correlation matrix composed by DCCA coefficients: evidence from the Chinese stock market. Physica A, 444, 667-679. http://dx.doi.org/10.1016/j.physa.2015.10.065.

Uryasev, S. (2000). Conditional value-at-risk: Optimization algorithms and applications. In Proceedings of the IEEE/IAFE/INFORMS 2000 Conference on Computational Intelligence for Financial Engineering (CIFEr) (Cat. No.00TH8520) (pp. 49-57). New York: IEEE. http://dx.doi.org/10.1109/CIFER.2000.844598.

Vercher, E., \& Bermúdez, J. D. (2015). Portfolio optimization using a credibility mean-absolute semi-deviation model. Expert Systems with Applications, 42(20), 7121-7131. http://dx.doi.org/10.1016/j.eswa.2015.05.020.

Yu, D., Wang, W., Zhang, W., \& Zhang, S. (2018). A bibliometric analysis of research on multiple criteria decision making. Current Science, 114(4), 747. http://dx.doi.org/10.18520/cs/v114/i04/747-758.

Zadeh, L. A. (1965). Fuzzy sets. Information and Control, 8(3), 338-353.

Zhang, W. G., \& Liu, Y. J. (2014). Credibilitic mean-variance model for multi-period portfolio selection problem with risk control. ORSpektrum, 36(1), 113-132. http://dx.doi.org/10.1007/s00291-013-0335-6.

Zhang, Y., Li, X., \& Guo, S. (2018). Portfolio selection problems with Markowitz's mean-variance framework: a review of literature. Fuzzy Optimization and Decision Making, 17(2), 1-34. http://dx.doi.org/10.1007/s10700-017-9266-z.

Zhao, L., Li, W., Fenu, A., Podobnik, B., Wang, Y., \& Stanley, H. E. (2018a). The q-dependent detrended cross-correlation analysis of stock market. Journal of Statistical Mechanics, 2018(2), 1-28. http://dx.doi.org/10.1088/1742-5468/aa9db0.

Zhao, L., Wang, G. J., Wang, M., Bao, W., Li, W., \& Stanley, H. E. (2018b). Stock market as temporal network. Physica A, 506, 11041112. http://dx.doi.org/10.1016/j.physa.2018.05.039. 